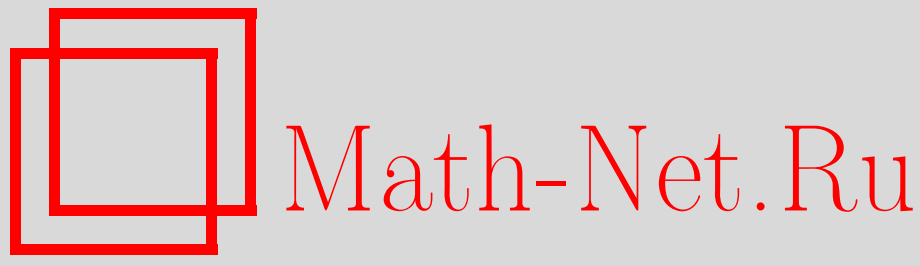

Обцероссийский математический портал

М. М. Маламуд, В. И. Могилевский, Обобщенные резольвенты изометрического оператора, Матем. заметки, 2003, том 73 , выпуск $3,460-465$

DOI: https://doi.org/10.4213/mzm618

Использование Общероссийского математического портала Math-Net.Ru подразумевает, что вы прочитали и согласны с пользовательским соглашением http://www.mathnet.ru/rus/agreement

Параметры загрузки:

IP : 18.234 .197 .8 
26 апреля 2023 г., 13:18:49

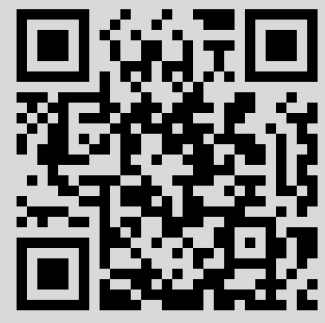




\section{ОБОБЩЕННЫЕ РЕЗОЛЬВЕНТЫ ИЗОМЕТРИЧЕСКОГО ОПЕРАТОРА}

\section{М.М. Маламуд, В.И. Могилевский}

1. Введение. В теории расширений симметрического оператора $A$ в гильбертовом пространстве $\mathfrak{H}$ хорошо известна следующая формула М. Г. Крейна для обобщенных резольвент оператора $A$ с равными дефектными числами (см., например, [1])

$$
\mathbb{R}_{\lambda}:=P_{\mathfrak{H}}(\widetilde{A}-\lambda)\left\lceil\mathfrak{H}=\left(A_{0}-\lambda\right)^{-1}-\gamma(\lambda)(\tau(\lambda)+M(\lambda))^{-1} \gamma^{*}(\bar{\lambda}), \quad \lambda \in \mathbb{C}_{ \pm}\right.
$$

Формула (1) устанавливает биективное соответствие между обобщенными резольвентами $\mathbb{R}_{\lambda}$ и оператор-функциями $\tau(\lambda)$ пополненного класса Неванлинны $\widetilde{R}_{\mathscr{H}}\left(\tau \in \widetilde{R}_{\mathscr{H}}\right)$. Здесь $\widetilde{A}=\widetilde{A}^{*}-$ расширение с выходом в $\widetilde{\mathfrak{H}} \supset \mathfrak{H}$, а $A_{0}$ - фиксированное расширение в $\mathfrak{H}$.

В [2] приведен простой вьвод формулы Крейна (1) и найдена ее связь с граничными тройками $\Pi=\left\{\mathscr{H}, \Gamma_{0}, \Gamma_{1}\right\}$ оператора $A^{*}$. Именно, в [2] показано, что $A_{0}=\operatorname{Ker} \Gamma_{0}, \gamma(\lambda):=\left(\Gamma_{0}\left\lceil\mathfrak{H}_{\lambda}\right)^{-1}-\right.$ $\gamma$-поле оператора $A, M(\lambda):=\Gamma_{1} \gamma(\lambda)$ - функция Вейля, соответствующая тройке $\Pi$, a $\mathbb{R}_{\lambda} g$ ние граничной задачи $\left(A^{*}-\lambda\right) f=g,\left\{\Gamma_{0} \mathbb{R}_{\lambda} g,-\Gamma_{1} \mathbb{R}_{\lambda} g\right\} \in \tau(\lambda)$.

$\mathrm{C}$ другой стороны, в работах [3], [4] (см. также библиограффию в [3]) получен аналог формулы Крейна для обобщенных резольвент изометрического оператора $V\left(V^{*} V=I\right)$.

Далее, в работе [5] нами получен аналог формулы Крейна для резольвент дуальной пары $\{A, B\}$ (напомним, что пару $\{A, B\}$ замкнутых линейных операторов $A$ и $B$ в $\mathfrak{H}$ называют дуальной парой (ДП), если $(A f, g)=(f, B g), f \in \mathscr{D}(A), g \in \mathscr{D}(B))$. Именно, упомянутая формула из [5] имеет вид (1) и устанавливает биективное соответствие между множеством $\Omega\left(\{A, B\}, \lambda_{0}\right\}$ обобщенных резольвент $\mathbb{R}_{\lambda}$ голоморфных в точке $\lambda_{0}$ и множеством голоморфных в точке $\lambda_{0}$ семейств $\tau$, для которых $0 \in \rho\left(\tau\left(\lambda_{0}\right)+M\left(\lambda_{0}\right)\right)$.

Заметим, что пара операторов $\{A, A\}$ является дуальной в точности тогда, когда $A$ симметричен $\left(A \subset A^{*}\right)$. Для этого случая упомянутая формула из [5] дополняет формулу Крейна и совпадает с ней для самосопряженных расширений $\widetilde{A}=\widetilde{A}^{*}$, если только граничная тройка $\Pi=\left\{\mathscr{H}, \Gamma_{0}, \Gamma_{1}\right\}$ такова, что $A_{i}:=\operatorname{ker} \Gamma_{i}=A_{i}^{*}, i=0,1$.

В настоящем сообщении мы приводим более общий, чем в [3], [4], вариант формулы для обобщенных $U$-резольвент ( $U$ унитарен в $\widetilde{\mathfrak{H}} \supset \mathfrak{H}$ ) изометрического оператора $V$ и показываем, что она 
также является следствием общей формулы из [5], примененной к ДП $\left\{V, V^{-1}\right\}$. Именно, каждая граничная тройка П ДП $\left\{V, V^{-1}\right\}$ порождает формулу обобщенных резольвент вида (1), a eе параметры $A_{0}, M, \gamma$ и $\tau$ выражаются, как и в симметрическом случае $(\{A, B\}=\{A, A\})$, через граничные операторы. Заметим, однако, что в случае ДП $\left\{V, V^{-1}\right\}$ аналогом класса Неванлинны, которому в симметрическом случае принадлежат $M$ и $\tau$, является класс Шура сжимающих в $\mathbb{D}:=\{\lambda \in \mathbb{C}:|\lambda|<1\}$ оператор-функций.

В связи с этим естественно возникает, оказавшаяся нетривиальной, задача описания граничных троек П дуальной пары $\left\{V, V^{-1}\right\}$, в которых обобщенным $U$-резольвентам соответствуют в формуле (1) оператор-функции $\tau(\lambda)^{-1}$, принадлежащие классу Шура в $\mathbb{D}^{e}:=\{z \in \mathbb{C}:|z|>1\}$. Мы назьваем такие тройки сжиммающими и описываем их (см. предложение 7).

Для этого случая из формулы вида (1) для ДП $\left\{V, V^{-1}\right\}$ мы получаем в $\mathbb{D}^{e}$ формулу

$$
\mathbb{R}_{\lambda}:=P_{\mathfrak{H}}(\widetilde{U}-\lambda)\left\lceil\mathfrak{H}=\left(A_{0}-\lambda\right)^{-1}+\gamma_{\Pi}(\lambda) K(\lambda)(I-M(\lambda) K(\lambda))^{-1} \gamma_{\Pi^{*}}^{*}(\bar{\lambda}), \quad \lambda \in \mathbb{D}^{e},\right.
$$

в которой функция Вейля $M(\lambda)$ строго сжимающая в $\mathbb{D}^{e}$. Здесь $\widetilde{U}-$ унитарное расширение с выходом в $\widetilde{\mathfrak{H}} \supset \mathfrak{H}$, а $A_{0}$ - фиксированное сжимающее расширение в $\mathfrak{H}$, причем $A_{0}\left\lceil\mathscr{D}(V)^{\perp}-\right.$ строгое сжатие, а $K(\lambda)=\tau(\lambda)^{-1}$ пробегает класс Шура в $\mathbb{D}^{e}$.

При этом так же, как и в [2], все параметры из формулы (2) выражаются через граничные операторы граничной тройки П дуальной пары $\left\{V, V^{-1}\right\}$.

Заметим еще, что совокупности сжатий $A_{0}:=\operatorname{ker} \Gamma_{0}^{V^{-1}}$ и функций Вейля $M(\lambda)$, соответствующих сжимающим граничным тройкам, описаны в следствии 9 и теореме 11.

В случае специальной граничной тройки $\Pi_{0}$ вида (8) формула (2) совпадает с таковой из [3], [4], а найденная связь с тройкой П 0 позволяет дополнить ее в некоторых аспектах.

2. Предварительные сведения. Пусть $\left[\mathfrak{H}_{1}, \mathfrak{H}_{2}\right]([\mathfrak{H}])$ - множество ограниченных операторов из $\mathfrak{H}_{1}$ в $\mathfrak{H}_{2}($ в $\mathfrak{H}), P_{L}$ - ортопроектор на подпространство $L$ в $\mathfrak{H}$. Введем следующие обозначения: $\widetilde{\mathscr{C}}\left(\mathfrak{H}_{1}, \mathfrak{H}_{2}\right)(\widetilde{\mathscr{C}}(\mathfrak{H}))$ - множество замкнутых линейных отношений (подпространств) в $\mathfrak{H}_{1} \oplus \mathfrak{H}_{2}$ $($ в $\mathfrak{H} \oplus \mathfrak{H}) ; T^{*} \in \widetilde{\mathscr{C}}\left(\mathfrak{H}_{2}, \mathfrak{H}_{1}\right)$ - отношение, сопряженное к $T \in \widetilde{\mathscr{C}}\left(\mathfrak{H}_{1}, \mathfrak{H}_{2}\right) ; \mathscr{D}(T), \mathscr{R}(T)$ и Кеr $T$ - область определения, образ и ядро отношения $T$ соответственно. Замкнутый оператор $T$ из $\mathfrak{H}_{1}$ в $\mathfrak{H}_{2}$ будем отождествлять с его графиком, так что $T \in \widetilde{\mathscr{C}}\left(\mathfrak{H}_{1}, \mathfrak{H}_{2}\right)$.

Пусть $\rho(T)=\left\{\lambda \in \mathbb{C}:(T-\lambda)^{-1} \in[\mathfrak{H}]\right\}-$ множество регулярных точек линейного отношения $T \in \widetilde{\mathscr{C}}(\mathfrak{H}), \mathfrak{N}_{T}:=\mathscr{D}(T)^{\perp}=T^{*}(0), \widehat{\mathfrak{N}}_{T}:=\{0\} \oplus \mathfrak{N}_{T} \subset T^{*}$. Обозначим через $\mathfrak{N}_{\lambda}(T)$ дефектное подпространство отношения $T$, т.е. $\mathfrak{N}_{\lambda}(T):=\mathfrak{H} \ominus \mathscr{R}(T-\bar{\lambda})=\operatorname{Ker}\left(T^{*}-\bar{\lambda}\right)$ и $\widehat{\mathfrak{N}}_{\lambda}(T):=\{\{f, \lambda f\}:$ $\left.f \in \mathfrak{N}_{\lambda}(T)\right\} \subset \mathfrak{H} \oplus \mathfrak{H}$.

Напомним, далее, некоторые определения и результаты из [5].

ОПРЕДЕЛЕнИЕ 1. Линейное отношение $\widetilde{A} \in \widetilde{\mathscr{C}}(\mathfrak{H})$ назьвается собственным расширением ДП $\{A, B\}$, если $A \subset \widetilde{A} \subset B^{*}$, т.е. $A \subset \widetilde{A}$ и $B \subset \widetilde{A}^{*}$. Множество собственных расширений ДП $\{A, B\}$ обозначим $\operatorname{Ext}\{A, B\}$.

ОПРЕДЕЛЕНИЕ 2. Совокупность $\Pi=\left\{\mathscr{H}_{0} \oplus \mathscr{H}_{1}, \Gamma^{B}, \Gamma^{A}\right\}$, в которой $\mathscr{H}_{0}$ и $\mathscr{H}_{1}-$ гильбертовы пространства, а $\Gamma^{B}=\left(\Gamma_{0}^{B}, \Gamma_{1}^{B}\right)^{\top}: B^{*} \rightarrow \mathscr{H}_{0} \oplus \mathscr{H}_{1}$ и $\Gamma^{A}=\left(\Gamma_{0}^{A}, \Gamma_{1}^{A}\right)^{\top}: A^{*} \rightarrow \mathscr{H}_{1} \oplus \mathscr{H}_{0}-$ линейные отображения, назьвается граничной тройкой ДП $\{A, B\}$, если $\Gamma^{B} B^{*}=\mathscr{H}_{0} \oplus \mathscr{H}_{1}$, $\Gamma^{A} A^{*}=\mathscr{H}_{1} \oplus \mathscr{H}_{0}$ и справедлива следующая формула Грина:

$$
\left(f^{\prime}, g\right)-\left(f, g^{\prime}\right)=\left(\Gamma_{1}^{B} \widehat{f}, \Gamma_{0}^{A} \widehat{g}\right)-\left(\Gamma_{0}^{B} \widehat{f}, \Gamma_{1}^{A} \widehat{g}\right), \quad \widehat{f}=\left\{f, f^{\prime}\right\} \in B^{*}, \quad \widehat{g}=\left\{g, g^{\prime}\right\} \in A^{*} .
$$

$\mathrm{C}$ каждой граничной тройкой $\Pi=\left\{\mathscr{H}_{0} \oplus \mathscr{H}_{1}, \Gamma^{B}, \Gamma^{A}\right\}$ ДП $\{A, B\}$ естественно связаны собственные расширения $A_{0}:=\operatorname{Ker} \Gamma_{0}^{B}$ и $A_{1}:=\operatorname{Ker} \Gamma_{1}^{B} \in \operatorname{Ext}\{A, B\}$. Справедливы следуюшие соотношения:

1) $\operatorname{Ker} \Gamma^{B}=A$ и $\operatorname{Ker} \Gamma^{A}=B$, причем $\Gamma^{B} \in\left[B^{*}, \mathscr{H}_{0} \oplus \mathscr{H}_{1}\right]$ и $\Gamma^{A} \in\left[A^{*}, \mathscr{H}_{1} \oplus \mathscr{H}_{0}\right]$;

2) совокупность $\Pi^{*}=\left\{\mathscr{H}_{1} \oplus \mathscr{H}_{0}, \Gamma^{A}, \Gamma^{B}\right\}$ является граничной тройкой для ДП $\{B, A\}$; 
3) отображение $\Gamma^{B}: \widehat{f} \rightarrow\left\{\Gamma_{0}^{B} \widehat{f}, \Gamma_{1}^{B} \widehat{f}\right\}\left(\widehat{f} \in B^{*}\right)$ задает биективное соответствие между множествами $\operatorname{Ext}\{A, B\}$ и $\widetilde{\mathscr{C}}\left(\mathscr{H}_{0}, \mathscr{H}_{1}\right): \widetilde{A} \rightarrow \Gamma^{B}(\widetilde{A})=: \theta \in \widetilde{\mathscr{C}}\left(\mathscr{H}_{0}, \mathscr{H}_{1}\right) ;$ полагаем $\widetilde{A}_{\theta}:=\widetilde{A}$, T.e.

$$
\widetilde{A}_{\theta}:=\left(\Gamma^{B}\right)^{-1} \theta=\left\{\widehat{f} \in B^{*}: \Gamma^{B} \widehat{f} \in \theta\right\}, \quad \theta \in \widetilde{\mathscr{C}}\left(\mathscr{H}_{0}, \mathscr{H}_{1}\right),
$$

при этом $\left(\widetilde{A}_{\theta}\right)^{*} \in \operatorname{Ext}\{B, A\}$ и $\left(\widetilde{A}_{\theta}\right)^{*}=\widetilde{A}_{\theta^{*}}\left(\right.$ для тройки $\left.\Pi^{*}\right)$;

4) если $B_{0}:=\operatorname{Ker} \Gamma_{0}^{A} \in \operatorname{Ext}\{B, A\}$ и $B_{1}=\operatorname{Ker} \Gamma_{1}^{A} \in \operatorname{Ext}\{B, A\}$, то $B_{0}=A_{0}^{*}$ и $B_{1}=A_{1}^{*}$.

Граничной тройке П сопостовляем $\gamma$-поле $\gamma_{\Pi}(\lambda):=\pi_{1}\left(\Gamma_{0}^{B} \uparrow \widehat{\mathfrak{N}}_{\lambda}(B)\right)^{-1}\left(\in\left[\mathscr{H}_{0}, \mathfrak{N}_{\lambda}(B)\right]\right)$, $\lambda \in \rho\left(A_{0}\right)$, где $\pi_{1}\left(\pi_{2}\right)$ - ортопроектор в $\mathfrak{H} \oplus \mathfrak{H}$ на первую (вторую) компоненту. В [5] показывается, что $\gamma_{\Pi}(\lambda)$ - голоморфная в $\rho\left(A_{0}\right)$ оператор-функция, удовлетворяюшая соотношению

$$
\gamma_{\Pi}(\mu)=\gamma_{\Pi}(\lambda)+(\mu-\lambda)\left(A_{0}-\mu\right)^{-1} \gamma_{\Pi}(\lambda), \quad \lambda, \mu \in \rho\left(A_{0}\right) .
$$

Аналогично граничной тройке $\Pi^{*}$ сопостовляется $\gamma$-поле $\gamma_{\Pi^{*}}(z)=\pi_{1}\left(\Gamma_{0}^{A} \uparrow \widehat{\mathfrak{N}}_{z}(A)\right)^{-1}\left(\in\left[\mathscr{H}_{1}\right.\right.$, $\left.\left.\mathfrak{N}_{z}(A)\right]\right), z \in \rho\left(A_{0}^{*}\right)$. Оператор-функция $\gamma_{\Pi^{*}}(z)$ голоморфна в $\rho\left(A_{0}^{*}\right)$.

ОПРЕДЕЛЕниЕ 3 [5]. Оператор-функция $M(\lambda):=M_{\Pi}(\lambda)$, определенная в $\rho\left(A_{0}\right)$ равенством $\Gamma_{1}^{B} \widehat{f}_{\lambda}=M(\lambda) \Gamma_{0}^{B} \widehat{f}_{\lambda}, \widehat{f}_{\lambda}=\left\{f_{\lambda}, \lambda f_{\lambda}\right\} \in \widehat{\mathfrak{N}}_{\lambda}(B), \lambda \in \rho\left(A_{0}\right)$, называется функиией Вейля, соответствующей граничной тройке П.

Оператор-функция $M(\lambda)$ голоморфна в $\rho\left(A_{0}\right)$, принимает значения в [ $\left.\mathscr{H}_{0}, \mathscr{H}_{1}\right]$ и удовлетворяет равенству

$$
M(\mu)-M(\lambda)=(\mu-\lambda) \gamma_{\Pi^{*}}^{*}(\bar{\lambda}) \gamma_{\Pi}(\mu), \quad \mu, \lambda \in \rho\left(A_{0}\right) .
$$

Кроме того, функция Вейля $M_{\Pi^{*}}(z), z \in \rho\left(A_{0}^{*}\right)$, соответствующая тройке $\Pi^{*}$, связана с $M_{\Pi}(\lambda)$ равенством $M_{\Pi^{*}}(\bar{\lambda})=M_{\Pi}^{*}(\lambda), \lambda \in \rho\left(A_{0}\right)$.

ПрЕДЛОЖЕНИЕ 4 [5]. Пусть П $=\left\{\mathscr{H}_{0} \oplus \mathscr{H}_{1}, \Gamma^{B}, \Gamma^{A}\right\}-$ әраничная тройка ДП $\{A, B\}$, $M(\lambda)$ - соответствуюшая функиия Вейля, $A_{0}:=\operatorname{Ker} \Gamma_{0}^{B}$ и $\widetilde{A}=\widetilde{A}_{\theta} \in \operatorname{Ext}\{A, B\}$. Тогда

1) $\lambda \in \rho\left(\widetilde{A}_{\theta}\right) \cap \rho\left(A_{0}\right)$ точно тогда, когда $0 \in \rho(\theta-M(\lambda))$;

2) справедлива следующая формула для канонических резольвент:

$$
\left(\widetilde{A}_{\theta}-\lambda\right)^{-1}=\left(A_{0}-\lambda\right)^{-1}+\gamma_{\Pi}(\lambda)(\theta-M(\lambda))^{-1} \gamma_{\Pi^{*}}^{*}(\bar{\lambda}), \quad \lambda \in \rho\left(A_{\theta}\right) \cap \rho\left(A_{0}\right) .
$$

ОПРЕДЕЛЕНИЕ 5. Отношением запрета, соответствующим граничной тройке П, называется линеал $\mathscr{F}_{\Pi}=\Gamma^{B} \widehat{\mathfrak{N}}_{B}=\left\{\left\{\Gamma_{0}^{B} \widehat{n}, \Gamma_{1}^{B} \widehat{n}\right\}: \widehat{n}=\{0, n\} \in \widehat{\mathfrak{N}}_{B}\right\} \subset \mathscr{H}_{0} \oplus \mathscr{H}_{1}$.

Дуальная пара $\{A, B\}$ назьвается ограниченной, если $A \in[\mathscr{D}(A), \mathfrak{H}]$ и $B \in[\mathscr{D}(B), \mathfrak{H}]$. Пусть $\Pi=\left\{\mathscr{H}_{0} \oplus \mathscr{H}_{1}, \Gamma^{B}, \Gamma^{A}\right\}$ - граничная тройка для ограниченной ДП $\{A, B\}$ такая, что $A_{0} \in[\mathfrak{H}]$. Тогда

1) корректно определены операторы

$$
\gamma_{\Pi}=\pi_{2}\left(\Gamma_{0}^{B} \uparrow \widehat{\mathfrak{N}}_{B}\right)^{-1} \in\left[\mathscr{H}_{0}, \mathfrak{N}_{B}\right], \quad \gamma_{\Pi^{*}}=\pi_{2}\left(\Gamma_{0}^{A} \uparrow \widehat{\mathfrak{N}}_{A}\right)^{-1} \in\left[\mathscr{H}_{1}, \mathfrak{N}_{A}\right] ;
$$

2) $\mathscr{F}_{\Pi} \in\left[\mathscr{H}_{0}, \mathscr{H}_{1}\right]$ и справедливо равенство

$$
M(\lambda)=\mathscr{F}_{\Pi}+\gamma_{\Pi^{*}}^{*}\left(A_{0}-\lambda\right)^{-1} \gamma_{\Pi}, \quad \lambda \in \rho\left(A_{0}\right) .
$$

3. Сжимающие граничные тройки. Пусть $V$ - изометрия в гильбертовом пространстве $\mathfrak{H}$ с областью определения $\mathscr{D}(V)$ и образом $\mathscr{R}(V)$. Положим $\mathfrak{N}=\mathfrak{N}_{V}\left(=\mathscr{D}(V)^{\perp}\right), \mathfrak{M}=\mathfrak{N}_{V^{-1}}$ $\left(=\mathscr{R}(V)^{\perp}\right)$. Так как $\left(V^{-1}\right)^{*}=\left(V^{*}\right)^{-1}$, имеет место эквивалентность $\left\{f, f^{\prime}\right\} \in\left(V^{-1}\right)^{*} \Longleftrightarrow$ $\left\{f^{\prime}, f\right\} \in V^{*}$. Пусть, далее, $C\left(\mathfrak{H}_{1}, \mathfrak{H}_{2}\right):=\left\{T \in\left[\mathfrak{H}_{1}, \mathfrak{H}_{2}\right]:\|T\| \leqslant 1\right\}-$ множество сжатий из $\mathfrak{H}_{1}$ в $\mathfrak{H}_{2}$, а $C_{0}\left(\mathfrak{H}_{1}, \mathfrak{H}_{2}\right):=\left\{T \in\left[\mathfrak{H}_{1}, \mathfrak{H}_{2}\right]:\|T\|<1\right\}$ - множество строгих сжатий; $C(\mathfrak{H}):=C(\mathfrak{H}, \mathfrak{H})$ и $C_{0}(\mathfrak{H}):=C_{0}(\mathfrak{H}, \mathfrak{H})$.

Обозначим $C(V):=\operatorname{Ext}\left\{V, V^{-1}\right\} \cap C(\mathfrak{H})$ - множество собственных сжимающих расширений ДП $\left\{V, V^{-1}\right\}$. Всякое сжимающее расширение $\widetilde{A}$ изометрии $V$ имеет вид $\widetilde{A}=\operatorname{diag}(V, T) \in$ $[\mathscr{D}(V) \oplus \mathfrak{N}, \mathscr{R}(V) \oplus \mathfrak{M}]$, где $T \in C(\mathfrak{N}, \mathfrak{M})$ - сжатие, и, значит, обязательно является собственным, $\widetilde{A} \in C(V)$. 
ОПРЕДЕЛЕниЕ 6. Граничную тройку П $=\left\{\mathscr{H}_{0} \oplus \mathscr{H}_{1}, \Gamma^{V^{-1}}, \Gamma^{V}\right\}$ ДП $\left\{V, V^{-1}\right\}$ назовем $с ж и$ мающ, еи, если верна эквивалентность

$$
\widetilde{A}=\widetilde{A}_{\theta} \in C(V) \Longleftrightarrow K:=\theta^{-1} \in C\left(\mathscr{H}_{1}, \mathscr{H}_{0}\right)
$$

Приведем критерий сжимаемости граничной тройки.

ПрЕДЛОЖенИЕ 7. Пусть П $=\left\{\mathscr{H}_{0} \oplus \mathscr{H}_{1}, \Gamma^{V^{-1}}, \Gamma^{V}\right\}-$ әраничная тройка ДП $\left\{V, V^{-1}\right\}$, $A_{0}=\operatorname{Ker} \Gamma_{0}^{V^{-1}}, \mathscr{F}_{\Pi}-$ запретный оператор. Тогда следующ,ие утверждения эквивалентHbl:

1) $\Pi=\left\{\mathscr{H}_{0} \oplus \mathscr{H}_{1}, \Gamma^{V^{-1}}, \Gamma^{V}\right\}-$ сжимающая граничная тройка;

2) $A_{0} \in C(V)$ u onepamop

$$
U=\left(\begin{array}{cc}
A_{0} & \gamma_{\Pi} \\
-\gamma_{\Pi^{*}}^{*} & \mathscr{F}_{\Pi}
\end{array}\right): \mathfrak{H} \oplus \mathscr{H}_{0} \rightarrow \mathfrak{H} \oplus \mathscr{H}_{1}
$$

унитарен;

3) справедливы равенства

$$
\Gamma_{1}^{V^{-1}}\left\{f, f^{\prime}\right\}=-\Gamma_{0}^{V}\left\{f^{\prime}, f\right\}, \quad \Gamma_{0}^{V^{-1}}\left\{f, f^{\prime}\right\}=-\Gamma_{1}^{V}\left\{f^{\prime}, f\right\}, \quad\left\{f, f^{\prime}\right\} \in\left(V^{-1}\right)^{*} .
$$

При выполнении каждого из условий 1)-3) функиия Вейля $M(\lambda)$, соответсвующая тройке П, является голоморфной и строго сжимающей в $\mathbb{D}^{e}:=\{\lambda \in \mathbb{C}:|\lambda|>1\}$.

СледствиЕ 8. Если П-сжимающая граничная тройка ДП $\left\{V, V^{-1}\right\}$, mо $A_{0} \uparrow \mathfrak{N} \in$ $C_{0}(\mathfrak{N}, \mathfrak{M})$. Обратно, если $\widetilde{A}_{0} \in C(V)$, причем $\widetilde{A}_{0}\left\lceil\mathfrak{N} \in C_{0}(\mathfrak{N}, \mathfrak{M})\right.$, то существует сжимающ, ая граничная тройка $\Pi_{0}=\left\{\mathscr{H}_{0} \oplus \mathscr{H}_{1}, \Gamma^{V^{-1}}, \Gamma^{V}\right\}$, для которой $\widetilde{A}_{0}=A_{0}:=\operatorname{Ker} \Gamma_{0}^{V^{-1}}$.

Примером сжимающей граничной тройки является тройка $\Pi_{0}=\left\{\mathfrak{N} \oplus \mathfrak{M}, \Gamma^{V^{-1}}, \Gamma^{V}\right\}$ вида

$$
\begin{aligned}
& \Gamma_{1}^{V^{-1}} \widehat{f}=-D_{0} P_{\mathfrak{N}} f+\left(A_{0}^{\prime}\right)^{*} D_{0 *}^{-1}\left(f^{\prime}-\widetilde{A}_{0} f\right), \quad \Gamma_{0}^{V^{-1}} \widehat{f}=D_{0 *}^{-1}\left(f^{\prime}-\widetilde{A}_{0} f\right), \\
& \Gamma_{1}^{V} \widehat{g}=-D_{0 *} P_{\mathfrak{M}} g+A_{0}^{\prime} D_{0}^{-1}\left(g^{\prime}-\widetilde{A}_{0}^{*} g\right), \quad \Gamma_{0}^{V} \widehat{g}=D_{0}^{-1}\left(g^{\prime}-\widetilde{A}_{0}^{*} g\right), \\
& \widehat{f}=\left\{f, f^{\prime}\right\} \in\left(V^{-1}\right)^{*}, \quad \widehat{g}=\left\{g, g^{\prime}\right\} \in V^{*},
\end{aligned}
$$

где $A_{0}^{\prime}:=\widetilde{A}_{0}\left\lceil\mathfrak{N}, D_{0}:=\left(I-\left(A_{0}^{\prime}\right)^{*} A_{0}^{\prime}\right)^{1 / 2} \in[\mathfrak{N}]\right.$ и $D_{0 *}:=\left(I-A_{0}^{\prime}\left(A_{0}^{\prime}\right)^{*}\right)^{1 / 2} \in[\mathfrak{M}]$.

Из предложения 7 и формулы (4) вытекает

Следствие 9. Пусть П $=\left\{\mathscr{H}_{0} \oplus \mathscr{H}_{1}, \Gamma^{V^{-1}}, \Gamma^{V}\right\}-$ сжимающая әраничная тройка ДП $\left\{V, V^{-1}\right\}$ и $M(\lambda)$ - coomветствуюшая функиия Вейля. Тогда

1) если $\widetilde{A}_{\theta} \in C(V)$, то $\widetilde{A}_{\theta}\left\lceil\mathfrak{N} \in C_{0}(\mathfrak{N}, \mathfrak{M})\right.$ точно тогда, когда $K:=\theta^{-1} \in C_{0}\left(\mathscr{H}_{1}, \mathscr{H}_{0}\right)$;

2) расширение $\widetilde{A}_{\theta} \in \operatorname{Ext}\{A, B\}$ унитарно точно тогда, когда оператор $\theta$ унитарен;

3) если $\widetilde{A}_{\theta} \in C(V)$, то формула (4) для канонических резольвент принимает вид

$$
\left(\widetilde{A}_{\theta}-\lambda\right)^{-1}=\left(A_{0}-\lambda\right)^{-1}+\gamma_{\Pi}(\lambda) K(I-M(\lambda) K)^{-1} \gamma_{\Pi^{*}}^{*}(\bar{\lambda}), \quad K:=\theta^{-1} .
$$

Так как для сжимающей граничной тройки П матрица $U$ вида (6) унитарна, то совокупность $\Delta=\left(A_{0}, \gamma_{\Pi},-\gamma_{\Pi^{*}}^{*}, \mathscr{F}_{\Pi}\right)$ образует унитарный узел с характеристической функцией $\theta(\lambda)=\mathscr{F}_{\Pi}+$ $\lambda \gamma_{\Pi^{*}}^{*}\left(\lambda A_{0}-I\right)^{-1} \gamma_{\Pi}(\lambda \in \mathbb{D})[6]$. Отсюда с учетом (5) вытекает 
ТЕорема 10. Пусть $\Pi=\left\{\mathscr{H}_{0} \oplus \mathscr{H}_{1}, \Gamma^{V^{-1}}, \Gamma^{V}\right\}-$ сжимающая граничная тройка ДП $\left\{V, V^{-1}\right\}$. Тогда соответствующая функиия Вейля $M(\lambda)$ голоморфна в $\mathbb{D}^{e}$, принимает значения в $C_{0}\left(\mathscr{H}_{0}, \mathscr{H}_{1}\right)$ и связана с характеристической функчией $\theta(\lambda)$ равенством $M(\lambda)=\theta\left(\lambda^{-1}\right)\left(\lambda \in \mathbb{D}^{e}\right)$. Обратно, для каждой голоморфной в $\mathbb{D}^{e}$ оператор-функции $M(\lambda)$ со значениями в $C_{0}\left(\mathscr{H}_{0}, \mathscr{H}_{1}\right)$ найдутся изометрия $V$ и сжимающая граничная тройка П для ДП $\left\{V, V^{-1}\right\}$ такие, что соответствующая функция Вейля $M_{\Pi}(\lambda)$ совпаdaem $c M(\lambda)$.

\section{4. Формула для обобщенных резольвент.}

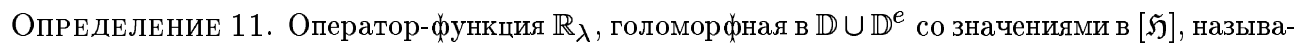
ется обобщенной $U$-резольвентой изометрического оператора $V$, если существуют гильертово пространство $\widetilde{\mathfrak{H}} \supset \mathfrak{H}$ и унитарный оператор $\widetilde{U}$ в $\widetilde{\mathfrak{H}}$ такие, что $V \subset \widetilde{U}$ и $\mathbb{R}_{\lambda}=P_{\mathfrak{H}}(\widetilde{U}-\lambda)^{-1} \uparrow \mathfrak{H}$, $\lambda \in \mathbb{D} \cup \mathbb{D}^{e}$.

В следующей теореме дается описание обобщенных $U$-резолвент изометрии $V$.

Теорема 12. Пусть $\Pi=\left\{\mathscr{H}_{0} \oplus \mathscr{H}_{1}, \Gamma^{V^{-1}}, \Gamma^{V}\right\}-$ сжимающая граничная тройка ДП $\left\{V, V^{-1}\right\}, A_{0}:=\operatorname{Ker} \Gamma_{0}^{V^{-1}}$ u $A_{1}:=\operatorname{Ker} \Gamma_{1}^{V^{-1}}=\left(A_{0}^{*}\right)^{-1}$. Пусть, далеe, $M(\lambda)=M_{\Pi}(\lambda)-$ соответствуюшая функиия Вейля, а $M_{\Pi^{*}}(\lambda)=M^{*}(\bar{\lambda})$ - функи, Вейля, соответствующ,ая тройке $\Pi^{*}=\left\{\mathscr{H}_{1} \oplus \mathscr{H}_{0}, \Gamma^{V}, \Gamma^{V-1}\right\}$ ДП $\left\{V^{-1}, V\right\}$. Тогда

i) $\mathbb{D}^{e} \subset \rho\left(A_{0}\right), \mathbb{D} \subset \rho\left(A_{1}\right)$ и формулы для обобщенных резольвент (в форме Крейна-Наймарка)

$$
\begin{gathered}
\mathbb{R}_{\lambda}=\left(A_{0}-\lambda\right)^{-1}+\gamma_{\Pi}(\lambda) K(\lambda)(I-M(\lambda) K(\lambda))^{-1} \gamma_{\Pi^{*}}^{*}(\bar{\lambda}), \quad \lambda \in \mathbb{D}^{e}, \\
\mathbb{R}_{\lambda}=\left(A_{1}-\lambda\right)^{-1}-\lambda^{-2} \gamma_{\Pi^{*}}\left(\lambda^{-1}\right) K^{*}\left(\bar{\lambda}^{-1}\right)\left(I-M_{\Pi^{*}}\left(\lambda^{-1}\right) K^{*}\left(\bar{\lambda}^{-1}\right)\right)^{-1} \gamma_{\Pi}^{*}\left(\bar{\lambda}^{-1}\right), \\
\lambda \in \mathbb{D},
\end{gathered}
$$

задалт биективное соответствие между мнохеством обобщенных U-резольвент $\mathbb{R}_{\lambda}$ и множеством голоморфных в $\mathbb{D}^{e}$ оператор-функиий $K(\lambda)$ со значениями в $C\left(\mathscr{H}_{1}, \mathscr{H}_{0}\right) ;$ при этом каноническим $U$-резольвентам $\mathbb{R}_{\lambda}$ и только им соответствуют в (10), (11) постоянные унитарнозначные оператор-функиии $K(\lambda)=$ : $K=\left(K^{-1}\right)^{*}\left(\right.$ последнее возмохно лишь тогда, когда $\left.\operatorname{dim} \mathfrak{N}_{0}=\operatorname{dim} \mathfrak{N}_{\infty}\right) ;$

ii) формулы для обобщенных резольвент (в форме А.В. Штрауса) имеют вид

$$
\mathbb{R}_{\lambda}= \begin{cases}\left(\widetilde{A}_{\tau_{1}(\lambda)}-\lambda\right)^{-1}, & \lambda \in \mathbb{D}^{e} \\ \left(\widetilde{A}_{\tau_{2}(\lambda)}-\lambda\right)^{-1}, & \lambda \in \mathbb{D}\end{cases}
$$

əде $\tau_{1}(\lambda):=K^{-1}(\lambda), \lambda \in \mathbb{D}^{e}, u \tau_{2}(\lambda):=K^{*}\left(\bar{\lambda}^{-1}\right), \lambda \in \mathbb{D}$

iii) для каждого $g \in \mathfrak{H}$ вектор-функиия $f:=\mathbb{R}_{\lambda} g$ является решением следуюшей әраничной задачи со спектральным параметром $K(\lambda)$ в граничном условии:

$$
\widehat{f}=\{f, \lambda f+g\} \in\left(V^{-1}\right)^{*}, \quad \begin{cases}\Gamma_{0}^{V^{-1}} \widehat{f}=K(\lambda) \Gamma_{1}^{V^{-1}} \widehat{f}, & \lambda \in \mathbb{D}^{e} \\ \Gamma_{1}^{V^{-1}} \widehat{f}=K^{*}\left(\bar{\lambda}^{-1}\right) \Gamma_{0}^{V^{-1}} \widehat{f}, & \lambda \in \mathbb{D} .\end{cases}
$$

ЗАмечАниЕ 13. Подчеркнем, что только обе формулы (10) и (11) вместе описьвают обобщенные $U$-резольвенты оператора $V$. Так, первая из них в отдельности описьвает лишь обобщенные резольвенты, порожденные всеми сжимающими расширениями, а вторая - теми растягивающими расширениями $\widetilde{A} \in \operatorname{Ext}\left\{V, V^{-1}\right\}$, для которых $0 \in \rho(\widetilde{A})$. 
ЗАмечание 14 . Пусть $\Pi=\left\{\mathscr{H}_{0} \oplus \mathscr{H}_{1}, \Gamma^{V^{-1}}, \Gamma^{V}\right\}$ - сжимающая граничная тройка ДП $\left\{V, V^{-1}\right\}$. Полагая $\Gamma_{0}:=\Gamma_{0}^{V^{-1}}=\pi_{0} \Gamma^{V^{-1}}$ и $\Gamma_{1}:=\Gamma_{1}^{V^{-1}}=\pi_{1} \Gamma^{V^{-1}}$, замечаем, что отображение $\Gamma=\left(\Gamma_{0}, \Gamma_{1}\right)^{\top}$ сюръективно. Далее, в силу (7) формула Грина (3) принимает вид

$$
\left(f^{\prime}, g^{\prime}\right)-(f, g)=\left(\Gamma_{0} \widehat{f}, \Gamma_{0} \widehat{g}\right)-\left(\Gamma_{1} \widehat{f}, \Gamma_{1} \widehat{g}\right), \quad \widehat{f}=\left\{f, f^{\prime}\right\} \in\left(V^{-1}\right)^{*}, \quad \widehat{g}=\left\{g, g^{\prime}\right\} \in\left(V^{-1}\right)^{*} .
$$

Положим $\gamma_{0}(\lambda):=\pi_{1}\left(\Gamma_{0} \uparrow \widehat{\mathfrak{N}}_{\lambda}\left(V^{-1}\right)\right)^{-1}, M_{0}(\lambda):=M(\lambda)\left(\lambda \in \mathbb{D}^{e}\right)$ и

$$
\gamma_{1}(\mu):=\pi_{1}\left(\Gamma_{1} \uparrow \widehat{\mathfrak{N}}_{\mu}\left(V^{-1}\right)\right)^{-1}, \quad \mu \in \mathbb{D} .
$$

Пусть, далее, $M_{1}(\mu)\left(\in\left[\mathscr{H}_{1}, \mathscr{H}_{0}\right]\right)$ определена равенством

$$
\Gamma_{0} \widehat{f}_{\mu}=M_{1}(\mu) \Gamma_{1} \widehat{f}_{\mu}, \quad \widehat{f}_{\mu}=\left\{f_{\mu}, \mu f_{\mu}\right\} \in \widehat{\mathfrak{N}}_{\mu}\left(V^{-1}\right), \quad \mu \in \mathbb{D}
$$

Тогда $\gamma_{0}(\lambda)=\gamma_{\Pi}(\lambda), \gamma_{1}(\mu)=-\mu^{-1} \gamma_{\Pi^{*}}\left(\mu^{-1}\right)$ и $M_{1}(\mu)=M_{\Pi^{*}}\left(\mu^{-1}\right)$. Отметим также, чтов формуле (11) линейное отношение $A_{1}\left(=\left(A_{0}^{*}\right)^{-1}\right)$ имеет вид $A_{1}=\operatorname{Ker} \Gamma_{1}$. Теперь формулы $(10),(11)$ можно записать в более симметричном виде:

$$
\begin{aligned}
& \mathbb{R}_{\lambda}=\left(A_{0}-\lambda\right)^{-1}-\lambda^{-1} \gamma_{0}(\lambda) K(\lambda)\left(I-M_{0}(\lambda) K(\lambda)\right)^{-1} \gamma_{1}^{*}\left(\bar{\lambda}^{-1}\right), \quad \lambda \in \mathbb{D}^{e}, \\
& \mathbb{R}_{\lambda}=\left(A_{1}-\lambda\right)^{-1}+\lambda^{-1} \gamma_{1}(\lambda) K^{*}\left(\bar{\lambda}^{-1}\right)\left(I-M_{1}(\lambda) K^{*}\left(\bar{\lambda}^{-1}\right)\right)^{-1} \gamma_{0}^{*}\left(\bar{\lambda}^{-1}\right), \quad \lambda \in \mathbb{D} .
\end{aligned}
$$

ЗАмечание 15 . Пусть $\Pi=\left\{\mathfrak{N} \oplus \mathfrak{M}, \Gamma^{V^{-1}}, \Gamma^{V}\right\}$ - сжимающая граничная тройка вида (8). Тогда $\gamma_{\Pi}(\lambda)=-\left(\widetilde{A}_{0}-\lambda\right)^{-1} D_{0 *}, \gamma_{\Pi^{*}}^{*}(\bar{\lambda})=-\left(\widetilde{A}_{0}^{*}-\bar{\lambda}\right)^{-1} D_{0}$ и

$$
M(\lambda)=\left(A_{0}\lceil\mathfrak{N})^{*}+D_{0} P_{\mathfrak{N}}\left(\widetilde{A}_{0}-\lambda\right)^{-1} D_{0 *}, \quad \lambda \in \mathbb{D}^{e} .\right.
$$

Теперь формула (10) принимает вид

$$
\mathbb{R}_{\lambda}=\left(\widetilde{A}_{0}-\lambda\right)^{-1}+\left(\widetilde{A}_{0}-\lambda\right)^{-1} D_{0 *} K(\lambda)(I-M(\lambda) K(\lambda))^{-1} D_{0} P_{\mathfrak{N}}\left(\widetilde{A}_{0}-\lambda\right)^{-1}, \quad \lambda \in \mathbb{D}^{e} .
$$

Формула (12) для обобщенных резольвент совпадает с таковой из [3], [4], где она получена другими методами. При этом порождаемая тройкой (8) и вытекающая из (11), аналогичная (12) формула для $\lambda \in \mathbb{D}$ в работах [3], [4] отсутствует. Заметим еще, что в формуле (11) $A_{1}=\left(A_{0}^{*}\right)^{-1}$ является, вообще говоря линейньм отношением, а не оператором.

\section{СПИСОК ЦИТИРОВАННОЙ ЛИТЕРАТУРЫ}

1. Крейн М. Г., Лангер Г. К. // Функцион. анализ и его прилож. 1971. Т. 5. № 3. С. 54-69. 2. Derkach V. A., Malamud M. M. // J. Func. Anal. 1991. V. 95. № 1. P. 1-95. 3. Langer H., Sorjonen P. // Ann. Acad. Sci. Fenn. A1. 1974. V. 561. P. 3-45. 4. Langer H., Textorius B. // Invariant Subspaces and Other Topics. 6th Oper. Theory Conf. Proc., 1982. P. 103-118. 5. Maламуд М. М., Могилевский В. И. // Докл. НАН Украины. 1997. №1. С. 30-37. 6. Бродский М. С. // УМН. 1978. Т. 33. № 4. С. 141-168. 7. Секефальви-Надь Б., Фояш Ч. Гармонический анализ операторов в гильбертовом пространстве. М.: Мир, 1970.

(М. М. Маламуд) Донецкий национальњый университет, Украина (В.И. Могилевский) Луганский государственньй педагогический университет, Украина 07.06.2002 E-mail: mdm@dc.donetsk.ua, magellan@gts.lg.ua 